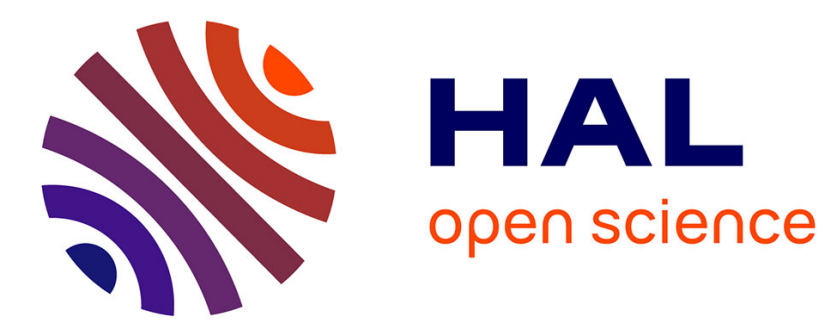

\title{
Contre-fictions : trois modes de combat Yves Citton
}

\section{To cite this version:}

Yves Citton. Contre-fictions : trois modes de combat. Multitudes, 2012, 58, pp.72-78. hal-00846472

\section{HAL Id: hal-00846472 \\ https://hal.science/hal-00846472}

Submitted on 24 Jul 2013

HAL is a multi-disciplinary open access archive for the deposit and dissemination of scientific research documents, whether they are published or not. The documents may come from teaching and research institutions in France or abroad, or from public or private research centers.
L'archive ouverte pluridisciplinaire HAL, est destinée au dépôt et à la diffusion de documents scientifiques de niveau recherche, publiés ou non, émanant des établissements d'enseignement et de recherche français ou étrangers, des laboratoires publics ou privés. 


\section{Majeure48 Contre-fictions politiques}

Campagnes publicitaires, propagandes soft et discours politiques nous racontent des histoires pour inciter à l'achat, au vote, à la conduite recherchée. Ne devons-nous pas trouver ou créer nos propres contre-fictions pour opposer de jolis coups de colère ou de beaux éclats de rire à ce storytelling et aux programmes les plus abrutissants du divertissement de masse? Ce dossier cherche à définir ces contre-fictions, multiples récits fictifs dont l'objet serait de transformer la réalité actuelle dans un projet de lutte contre la reproduction d'un donné perçu comme mutilant. 


\section{sommaire}

Réalités, fictions, contre-fictions... 70 Introduction

Contre-fictions: trois modes de combat

72 Yves Citton

Entre storytelling et contre-fictions 79 Insert 1

Contre-fictions de soi 81 Pascal Houba

Agencements contre-fictionnels de littérature collective

92 Insert 2

Charismes du réel 94 Pierangelo Di Vittorio

Fendre le possible 103 Insert 3 (Action30)
Contr(ôl)e-fiction : de l'Empire à l'Interzone 106 Frédéric Claisse

Dollhouse-Bildungsroman et contre-fiction 118 Insert 4 (Sandra Laugier)

Fictions et contre-fictions de l'âge du cyborg 121 Ariel Kyrou

This Spartan Life, un machinima au Congrès 132 Insert 5 (Isabelle Arvers)

L'imagination crépusculaire 135 Frédéric Neyrat

Graffitis contre-fictionnels 145 Insert 6 


\section{Introduction}

\section{Réalités, fictions, contre-fictions...}

Les fictions nous gouvernent de leur implacable réalité.

Les Romains avaient leur louve, leurs haruspices, leurs empereurs divinisés, leur apologue des membres et de l'estomac. Nous avons notre Marianne, nos taux de croissance du PIB, nos réalités augmentées et nos triples A de la finance.

Depuis la fin du XVII e siècle, toute une modernité critique s'est ingéniée à dénoncer ces fictions comme des «fables», et à vouloir les remplacer par «la vérité» (scientifique). De Spinoza à Bruno Latour, toute une altermodernité a cependant souligné la nécessité fonctionnelle de ces fables. Il ne suffit pas d'en déboulonner les machines pour en abolir les causes. Nul agir humain ne peut se passer de recourir à ce type de "faitiches».

C'est du côté de cette altermodernité que se situe ce dossier de Multitudes, coordonné par Pascal Houba et Ariel Kyrou. Fictions, fables, simulacres, storytelling sont certes voués à nous illusionner, à nous «raconter des histoires». Le problème ne vient toutefois pas tant de ces leurres que de leurs messages et du contenu spécifique auquel ils nous font accroire, ainsi que de la manière dont ils nous les fourguent. Plutôt qu'à se réclamer de la "vérité» contre la fiction, essayons de démêler ce qu'il y a de pernicieux et ce qu'il y a de salutaire dans la nécessaire fictionnalité des réalités humaines.

Se réclamer des vertus positives de la fiction devient un lieu commun de notre époque écrasée sous la chape de plomb d'un « réalisme» politico-économique qui constitue lui-même la plus massive et la plus néfaste de nos fables actuelles. Qu'on revendique ses propres «délits de fiction», qu'on théorise la puissance d'un «fictionnaire» à inventer, qu'on analyse savamment les enjeux des «mondes possibles» ou des fonctions anthropologiques de la fictionnalité ${ }^{1}$, nous sentons l'urgence de concrétiser en d'autres fictions ce cri selon lequel un «autre monde est possible». Sauf que la plupart d'entre nous s'époumonent à répéter ce même cri sans parvenir à le faire déboucher sur autre chose qu'un vœu pieux - une vaine fiction. Est-ce par manque de lucidité face à la puissance de confusion mais aussi d'habile divertissement des fables qui dessinent les contours béats de notre soumission? Comment, dès lors, nous repérer dans la jungle de nos fictions, dont on ne sait si elles nous appartiennent ou si elles ont été plantées discrètement dans notre cuir chevelu? Comment choisir, s'inspirer, créer et faire partager les fictions les plus justes et décapantes de cet «autre monde»?

Ce dossier tente de répondre à ces questions en proposant un terme, celui de contre-fictions, et en essayant d'en décliner quelques modes d'existences possibles, au point de jointure entre l'imaginaire narratif et l'activisme politique. On y définit une contre-fiction politique comme une unité narrative de dimensions et de substrats variables, allant du graffiti au récit littéraire, en passant par le canular et le détournement de jeu vidéo, qui introduirait dans la réalité un décalage fictionnel ayant pour finalité ou pour effet d'enrayer la reproduction systémique de cette réalité, afin d'en bloquer ou d'en infléchir le cours.

À travers une série d'articles de fond et d'inserts illustratifs, en alternance les uns avec les autres, ce dossier envisagera les contre-fictions du point de vue de leur rapport au réel, à l'éthique de la vérité, à l'auto-fiction, au storytelling, à l'activisme politique, au populisme, aux sociétés de contrôle, au capitalisme ou à nos nouvelles technologies dites d'augmentation de l'humain. Dans tous les cas, et sur tous ces domaines très variés, il s'agira d'opérer un même triple mouvement: mesurer la part des fictions sur lesquelles repose la reproduction de nos réalités actuelles; repérer les tentatives de contre-fictions qui prennent déjà leur contre-pied; mieux comprendre en quoi consiste la puissance virtuelle de ces contre-fictions afin d'en augmenter le nombre et le rayonnement.

Notre présent et notre avenir ont moins besoin de fictions en tant que telles que de changements de cap. Or y a-t-il de meilleur méthode, de meilleur jeu, de meilleur art pour réinventer le réel que de «contre-fictionner» via de multiples formes et supports la domination actuelle d'un capitalisme prédateur et suicidaire?

Les contre-fictions sont les poissons pilotes, dans et en dehors de nos têtes, de cet autre monde que nous cherchons à rendre possible. 1 Voir par exemple, pour s’en tenir aux publications les plus récentes: revue Vacarme, «Fictions à l'oeuvre» nº 54
(hiver 2011); Francis Jenvrey, Théorie du fictionnaire, Paris, Questions théoriques, 2011; Luc Lang, Délit de fiction. La littérature, pourquoi?, Paris, Folio, 2011; Françoise Lavocat (éd.), La théorie littéraire des mondes possibles, Paris,
CNRS Éditions, 2010; Anne Duprat et Francoise Lavocat (éd.), Fiction et cultures, Paris, SFLGC, 2010. 


\section{Contre-fictions: trois modes de combat Yves Citton}

En première approche, on peut définir une contre-fiction comme un récit fictionnel visant à transformer la réalité actuelle dans un projet de lutte contre la reproduction d'un donné perçu comme mutilant ${ }^{1}$.

La thèse sous-jacente est que le non-lieu de la fiction constitue un opérateur de déblocage devenu central dans les conflits politiques contemporains, en ce qu'il permet d'ouvrir un espace de rejet des données, dans un monde où ce sont de plus en plus les «données» qui nous oppriment. Par «données», on vise ici à la fois les data collectées, les discours d'experts qui mobilisent ces data pour nous enfermer dans l'existant, mais aussi les produits de consommation à bon marché qui nous sont «donnés» (vendus à trop vil prix). Le passage par la fiction devrait dès lors être assumé comme un passage nécessaire pour toute politique émancipatrice, qui comporterait inévitablement une dimension «visionnaire». On parlera de contre-fictions pour désigner des récits anti-systémiques dont la visée (ou l'effet) consiste à faire entrevoir un autre monde possible, afin de nous décoller des fausses évidences à travers lesquelles se reproduisent les données qui nous aveuglent et qui nous paralysent.

Apparaît ici un premier type de contre-fictions qui mériteraient d'être qualifiées d'initiatrices, en ce qu'elles tendent à dépeindre des mondes irréels, auxquels on donne une première forme d'existence (imaginaire) en permettant à des lecteurs, auditeurs ou spectateurs de s'y immerger à l'occasion d'un roman ou d'un spectacle. C'est ici le domaine revendiqué par le geste utopique: la production de croyances et de désirs effectivement conçus durant cette immersion fictionnelle constitue une forme de contre-conduite, en ce qu'elle nous pousse à désirer et à œuvrer pour la réalisation de l'autre monde possible qui s'y trouve dépeint.

1 Précisons que notre objet (essentiellement politique) est très différent des définitions techniques que les narouvrir dans le récit une branche narrative qui n'accède pas au statut de fait avéré». Voir sur ce point l'article «Contrefiction» rédigé par Maxime Abolgassemi pour l'Atelier de Théorie Littéraire du site Fabula (www.fabula.org/atelier. hp?Contrefiction).
Cette première approche mérite toutefois d'être précisée et corrigée sur plusieurs points, qui conduiront à redéfinir à la fois la nature, la présence et l'efficience des (contre-)fictions dans le monde contemporain.

Les choses qui ont été faites

1. La réalité actuelle se reproduit à travers la circulation d'une énorme quantité de fictions quotidiennes. Les fables économistes que j'entends à la radio durant le Journal du matin, le roman que je lis dans le métro, les petits mensonges que j'invente pour échapper à une obligation astreignante, le récit journalistique d'un mariage princier, la série télévisée du soir - autant de fictions, c'est-à-dire autant d'histoires que nous nous racontons, dont nous pouvons suspecter que le statut référentiel est sujet à caution, mais qui n'en canalisent pas moins la façon dont nous donnons collectivement sens au monde.

2. Parmi ces histoires que nous nous racontons, les unes (le roman, la série télévisée) s'affichent comme ne se référant pas à des faits observables dans la réalité actuelle, mais comme dépeignant des situations imaginaires, qui n'ont d'existence originelle que dans le cerveau de leurs créateurs. Les autres (les calculs de PIB, les excuses mensongères, le mariage princier) prétendent au contraire correspondre de façon adéquate à un état de choses réel. On réserve généralement le terme de "fiction» pour la première catégorie, tandis qu’on applique ceux de «vérité» ou de «mensonge» à la seconde.

3. Il convient toutefois de récuser cette distinction trop superficielle. Même en laissant de côté le cas du mensonge intentionnel, les prétentions de tout discours «véridique» (et sincère) à exprimer la réalité du monde actuel a depuis longtemps été dénoncée comme problématique. Les «faits» et les «données» sont précisément ce que nous dit l'étymologie: des facta et des data, soit des «choses qui ont été faites» et des "choses qui ont été données» - faites et données par quelqu'un, dans un certain but, sur la base d'une certaine sensibilité, à partir d'une certaine perspective, au sein d'un certain contexte, etc. Nous le savons tous: le PIB et le mariage princier sont des «choses qui ont été faites», "construites» selon une certaine grammaire, un certain vocabulaire, une certaine sémantique, une certaine axiologie qui leur donnent le sens que nous y trouvons.

\section{Facta et ficta}

4. Cette (nécessaire) suspicion constructiviste ne saurait toutefois confondre toutes les « données» dans une fictionnalité généralisée et indistincte. Il convient au contraire de préciser les problèmes en distinguant deux gestes de nature différente. On peut ainsi réserver le terme de fait pour désigner une présentation qui $\left(1^{\circ}\right)$ «construit» certes une réalité actuelle en un factum, en l'isolant du sein d'un continuum (selon certaines finalités, perspectives, valeurs, stratégies, etc.), mais qui $\left(2^{\circ}\right)$ peut se contenter de sélectionner cette partie de réalité pour la laisser ensuite se manifester d'elle-même, à travers des dispositifs d'enregistrement et de communication purement mécaniques. 
5. Prenons-en pour exemple un film documentaire montrant la cérémonie de mariage du 29 avril 2011 entre le prince William d'Angleterre et Kate Middleton, à l'aide d'une caméra fixe postée frontalement aux deux époux en train de prononcer leurs vœux. Bien entendu, le choix de considérer comme quelque chose d'«important» (et digne de Mondovision) les noces de William et Kate relève d'une énorme "histoire» que toute une misérable machine médiatique parvient remarquablement bien à nous fourguer selon une logique qui relève du pur «spectacle». Il ne serait pas faux de dire qu'on est ici dans la pure fiction, aussi loin que possible de la «réalité vraie» (qui serait celle des destructions environnementales, des viols du Congo, des mobilisations révolutionnaires de la Tunisie, etc.). Résistons pourtant à cette tentation, et obstinons-nous à définir étroitement «le fait » par la présentation d'une réalité qui se manifeste d'elle-même, dès lors qu'on y expose des appareils mécaniques d'enregistrement.

6. Réservons le terme de fiction, plus étroitement défini, pour la présentation sensorielle de réalités qui ne se laissent pas enregistrer ou communiquer par des dispositifs mécaniques. Aux facta, «choses qui ont été faites» par un travail de sélection et d'isolation au sein de ce que «donne» à percevoir le monde actuel (avec toutes les histoires qui le structurent et le travestissent incessamment), on opposera les ficta, définies comme des «images qui ont été fabriquées» parce que le monde actuel ne fournit pas par lui-même des présentations qu'il suffirait d'enregistrer pour les mettre en circulation. Le latin fingere (dont dérivent ficta et fictio) désigne en effet le geste de "fabriquer, façonner, forger», tel que l'illustrent le peintre ou le sculpteur qui donnent forme à une matière visible (pigments, plâtre, granit) pour présenter une réalité invisible (Apollon, la Victoire, la colère). Les ficta sont toujours de nature allégorique: ils montrent quelque chose (de visible) pour faire percevoir autre chose (d'invisible comme tel).

7. L'opposition entre les facta et les ficta rejoint le réalisme (souvent disqualifié comme naï) défendu par André Bazin dès la fin des années 1940, lorsqu'il saluait la révolution esthétique, psychologique, médiologique et ontologique constituée par l'avènement de la photographie: "pour la première fois, entre l'objet initial et sa représentation, rien ne s'interpose qu'un autre objet. Pour la première fois, une image du monde extérieur se forme automatiquement, sans intervention créatrice de l'homme, selon un déterminisme rigoureux $»^{2}$. Filmé selon le dispositif évoqué plus haut, le mariage de William et de Kate appartient au domaine des facta: il a bien entendu été programmé (comme un spectacle), mais l'image photographique qui l'enregistre «automatiquement», analogiquement, présente une certaine réalité concrète «sans intervention créatrice de l'homme». Au contraire, le PIB relève du domaine des ficta: il nous donne une représentation «allégorique» de l'économie, de sa croissance ou de sa récession, lesquelles sont réputées échapper à la perception sensible.

Lorsque Jean Baudrillard nous décrit plongés dans un monde de simulations et de simulacres, lorsque Jonathan Beller anatomise notre «mode cinématographique de production ${ }^{3}$, ils décrivent un univers intégralement médiatisé où triomphent les fictions selon

André Bazin, «Ontologie de l'image photographique» in Qu'est-ce que le cinéma?, Paris, Éditions du Cerf, 2008, p. 13 3 Jean Baudrillard, Simulacre et simulation, Paris, Galilee, 1981; Jonathan Beller, The Cinematographic Mode of Pro-
duction. Attention Economy and The Society of the Spectacle, Hanover, Dartmouth UP, 2006. les deux sens esquissés ci-dessus. Nos «données» (supposées «réelles») sont soit des facta qui ont été mis en place par des programmations relevant du spectacle et du consumérisme capitaliste (comme le mariage princier), soit, pire encore, des ficta allégoriques dont c'est non seulement l'existence mais aussi le mode de présentation qui relèvent de la programmation (comme le PIB ou l'image digitale recomposée par Photoshop).

\section{Saborder le triomphe des fictions}

8. En recadrant l'histoire du cinéma dans la perspective des nouveaux médias numériques qui sont en train de reconfigurer nos perceptions du monde, Lev Manovich démontre qu'avec la multiplication et l'omniprésence des effets spéciaux permis par la digitalisation, «le cinéma numérique est une animation d'un type particulier qui utilise, entre autres nombreux éléments, le tournage en vues réelles ». Autrement dit, le cinéma de la dernière décennie s'est rapidement éloigné du modèle bazinien de l'ontologie photographique pour revenir aux premiers essais de peinture sur image et d'animation qui avaient été tentés à l'époque de Méliès: «issu de l'animation, le cinéma marginalisa celle-ci pour en devenir finalement un cas particulier $»^{4}$. Les facta de l'époque photographique, qui ont dominé le $\mathrm{XX}^{\mathrm{e}}$ siècle, sont désormais noyés dans les flots de ficta dont nous abreuve la fabrication d'images numérisées. En notre époque de simulation, les fictions du peintre (muté en animateur) dominent complètement les faits du photographe (toujours suspecté de recourir à Photoshop).

9. Ce triomphe de la fiction-simulation peut engendrer au moins deux types de réactions chez ceux qui ne se satisfont pas de la reproduction de l'ordre dominant. Certains ont choisi de se plonger dans le virtuel pour investir les potentiels subversifs offerts par un monde de simulation. Tels sont les Yes Men, qui profitent du flou des identités digitales pour emprunter des images de notoriété publique et pour les refaçonner provisoirement de l'intérieur afin de les décrédibiliser durablement envers l'extérieur ${ }^{5}$. Tels sont les divers films d'anticipation qui mobilisent l'imaginaire de la science-fiction et la machinerie des effets spéciaux numériques pour nous immerger dans un monde virtuel aussi convaincant qu'haïssable, dont on ne ressort que pour mieux s'apercevoir qu'il ressemble terriblement au nôtre. 10. Qu'ils jouent sur le rire ou sur l'horreur, de tels dispositifs donnent l'exemple d'un deuxième type de contre-fictions, qui peuvent être qualifiées de dénonciatrices: lecteurs et spectateurs s'y trouvent immergés dans un monde de ficta programmés, qui n'est investi que pour révéler sa ridicule ou intolérable vacuité. Le triomphe des fictions est contré par une stratégie de sabordage: le monde actuel, intégralement fictionné par les romans de gare, les séries télévisées, les mariages princiers, les spots publicitaires, les effets spéciaux et les calculs de PIB, se voit revisité avec des lunettes qui en font apparaitre la nature de «simulacre effectivement réel» - ce dont on espère induire des réactions de détachement (toujours relatif), voire de révolte, ou du moins de lucidité.

4 Lev Manovich, Le langage des nouveaux média (2001), Paris, Les Presses du réel, 2010, p. 520 5 Sur les Yes Men, voir Mike Bonanno \& Andy Bichlbaum, Les Yes Men. Comment démasquer (en s’amusant un peu)
limposture néolibérale, Paris, La Découverte, 2005. 
Résister par la vertu des faits

11. Une autre réaction possible au triomphe des fictions à l'âge des simulacres animés et des nouveaux médias consiste au contraire à tenter de retrouver la «charge de réalité » dont sont porteurs les facta enregistrés de façon analogique. Un troisième type de contre-fictions, sans doute le plus radical, peut être qualifié de documentaire, en ce qu'il s'efforce de contrer la fictionnalisation de notre monde actuel en enregistrant des «blocs de réalité » dont c'est la présentation mécanique qui nous fait redécouvrir un autre monde possible. Ce serait l'œuvre cinématographique de Jean-Marie Straub et Danièle Huillet qui pourrait servir d'emblème aux contre-fictions documentaires ${ }^{6}$. "On apprend du micro, en tournant en son direct, à entendre objectivement, et à forcer aussi le spectateur à entendre objectivement »; la prise de son directe permet «d'avoir des surprises, de découvrir une réalité, d'essayer des combinaisons, qui sont beaucoup plus riches que celles qu'on pourrait trouver soi-même, avec ses petites intentions». «L'unique devoir pour quelqu'un qui fait des films est de ne pas falsifier la réalité et d'ouvrir les yeux et les oreilles des gens avec ce qu'il y a, avec la réalité » ${ }^{7}$.

12. Dans ce troisième cas, comme le théorisait Gilles Deleuze dix ans après ces propos de Jean-Marie Straub, la résistance à la reproduction mutilante du « donné» implique une guerre contre les «clichés», dans laquelle l'arme principale est à chercher dans l'enregistrement photographique de ce que la réalité donne à percevoir (mais que les clichés effacent de nos perceptions). Le problème des ficta issus de l'activité de fabrication de quelque créateur humain que ce soit, c'est qu'ils ont toujours été pré-filtrés par les clichés dont la circulation colonise toutes nos têtes. En ce sens, le retour du cinéma à la dominance de l'animation (à travers la programmation numérique) sanctionne le triomphe du cliché: les «créateurs», auxquels une technologie de plus en plus souple permet de tout soumettre au contrôle de leur imagination, tendent à devenir les vecteurs transparents de la pure circulation des clichés. La programmation intégrale de tous les paramètres de l'image selon les intentions des producteurs abolit tout ce que la résistance du réel insufflait de vie (de «surprise», de «découverte») dans un film. Pour échapper à cela et défier la malléabilité du programme par la fixité de l'empreinte, la contre-fiction documentaire s'efforce de détruire l'intention: «j'essaie de ne pas avoir d'intention quand je tourne un film. Dès que je remarque qu'une intention apparaît derrière quelqu'arbre que ce soit, alors je la détruis $»^{8}$.

\section{Documenter la contre-fiction}

13. Contrairement à ce qu'Arturo Mazzarella critique dans un livre récent (où il prend pour cible les « non-fiction novels» à succès comme Gomorra de Roberto Saviano) ${ }^{9}$ Straub et Huillet n’ont jamais cru que la réalité se raconte d'elle-même ou qu'il suffise de planter une caméra

6 Ces réflexions ont été élaborées en collaboration étroite avec Philip Watts, dans le cadre d'un livre commun en préparation sur Le grain du réel, centré sur le cinéma de Jean-Marie Straub et Danièle Huillet.

août-septembre 1970, p. 53.

Id. p. 57.

9 Arturo Mazzarella, Politiche delli irrealtà. Scritture e visioni tra Gomorra e Abu Ghraib, Torino, Bollati Boringhieri, 2011. devant des cuistres couronnés pour en faire un document digne d'intérêt. Tout au contraire, leur cinéma consiste à faire sentir la matérialité la plus concrète de la mise en scène de contrefictions empruntées à Sophocle, Corneille, Hölderlin, Schönberg, Pavese, Fortini ou Vittorini. Qu'ils filment l'opéra Moïse et Aron ou qu'ils entraînent minutieusement des villageois italiens à déclamer des textes sur les coopératives ouvrières de l'après-guerre, ce n'est jamais directement la réalité donnée qu'ils cherchent à saisir par le mécanisme de leur caméra. C'est une réalité déplacée sur le terrain d'une fiction, une réalité re-façonnée par un texte littéraire, une réalité qui se reconstitue par elle-même devant leur appareil enregistreur au sein d'un dispositif fictionnel par lequel ils la pressurisent. Ce que documentent leurs contre-fictions, c'est cette reconstitution de la réalité en temps réel, riche de surprises et de résistances qui dépassent toutes les «petites intentions» des producteurs et des programmateurs.

14. Les contre-fictions documentaires visent donc à réagencer le rapport entre ficta et facta. Elles nous font voir que les facta sont des fictions, en mettant en scène un opéra, une tragédie ou un texte littéraire. Elles nous branchent sur la charge de réalité propre aux facta (que désamorce la programmation inhérente aux ficta): il faut enregistrer la mise en scène de la tragédie par un mécanisme analogique (en son direct), parce que ce sont les nécessités et les contingences mécaniques du réel (et non les clichés colonisant nos esprits) qu'il faut donner à voir. En nous «surprenant» par la découverte d'autres histoires-images-sons possibles, non-filtrés par les clichés et les programmes qui pré-conditionnent nos allégories, les contre-fictions documentaires sont profondément ancrées dans l'expérience moderniste, dont elles poursuivent un travail qui s'y faisait au nom de «l'écriture» (intransitive). Leur lutte contre le capitalisme-spectacle est toutefois plus radicale. Cette lutte se fonde sur une prémisse étroitement matérialiste: ce qui ne peut être enregistré par des machines n'existe pas. La vertu de cette attitude tient aux questions éminemment concrètes qu'elle invite à poser: peut-on filmer le PIB, la croissance, la démocratie? Que nous apprendrait le fait d'essayer de les filmer en son direct, sans recours aux ficta? Toute une révolution esthétique, épistémologique, éthique et politique est prête à irradier à partir de telles questions.

Trois modes de combat

15. Les trois types de contre-fictions esquissés ici relèvent de stratégies très différentes, mais s'inscrivent tous dans un même effort de lutte contre la prégnance de clichés qui paralysent la production de nouvelles émancipations possibles. Les contre-fictions initiatrices mènent cette lutte en frayant les voies d'un imaginaire alternatif, où l'on découvre de nouvelles façons de rêver un monde meilleur, moins pressurisé par les fausses urgences de la logique capitaliste. Les contre-fictions dénonciatrices investissent la fictionnalité inhérente à notre société du spectacle, pour faire imploser quelques-uns des référents creux auxquels se raccroche notre désorientation productiviste, en passe de sombrer dans le sécuritarisme suicidaire. Les contre-fictions documentaires vont chercher dans l'altérité de la réalité matérielle concrète de quoi surprendre et décoincer les limitations inhérentes à notre imagination intentionnelle indécrottablement colonisée par la nécessaire circulation des clichés. Plutôt que des genres 
(exclusifs entre eux), ces trois types de contre-fictions sont plutôt à concevoir comme trois modes de combat, qui peuvent parfois se superposer voire fusionner - comme l'illustrerait l'admirable Humiliés-Umiliati de Jean-Marie Straub et Danièle Huillet (2002).

Bruno Latour rappelait dans un numéro récent de Multitudes qu' «il n'y a pas de monde commun, qu'il n'y en a jamais eu », qu' «il est à composer» et que «si le monde commun est à composer, on peut rater sa composition $»^{10}$. Des trois modes de combat esquissés ci-dessus, et de la production contre-fictionnelle des années à venir, dépendra la composition d'un monde commun possible - ou son ratage.

\section{Insert 1}

\section{Entre storytelling et contre-fictions: l'activisme mystificateur}

Le storytelling est à la mode. Depuis le best-seller de Christian Salmon consacré à la façon dont managers, politiciens et publicitaires nous «racontent des histoires» pour mieux exploiter notre force de travail, capter nos intentions de vote ou nos préférences de consommation, la méfiance règne envers des machines narratives identifiées à des «machines à formater nos esprits $»^{1}$.

Cette dénonciation repose sur deux prémisses largement partagées. D’une part, on accuse les histoires racontées d'être des fables «leurrantes», faites pour travestir la réalité; d'autre part, on les accuse de caresser dans le sens du poil le système dominant (consumériste, capitaliste, néolibéral, sécuritaire, etc.), et donc de jouer pleinement le jeu de «la société du spectacle». Sous cette lumière accusatrice, le storytelling nous noie dans un bouillon d'illusions trompeuses, parfaitement alignées sur la reproduction ou l'exacerbation de notre oppression. À première vue, les contre-fictions échappent à cette définition péjorative du storytelling, en ce qu'elles visent à court-circuiter la reproduction des logiques oppressives qui nous aliènent (s'opposant frontalement à la seconde prémisse évoquée ci-dessus). Le statut de la fictionalité est toutefois plus complexe, et pourrait permettre des rapprochements suggestifs entre storytelling et contre-fictions.

Relevons d'abord que, dans le monde anglo-saxon, le storytelling est analysé non seulement comme un moyen de faire gober des salades aliénantes, mais également comme un outil d'activisme politique. Des syndicalistes mobilisent la force des récits pour aider des employés de maintenance latinos à raconter l'histoire de leur exploitation, de leur résistance et de leur lutte victorieuse, donnant ainsi à leur expérience exemplaire une force de rayonnement capable de se répandre comme une fièvre contagieuse. On peut multiplier les exemples d'un tel storytelling contre-systémique, dont les histoires sont faites pour catalyser des résistances à l'exploitation ainsi que des inventions de micropolitiques alternatives ${ }^{2}$.

1 Christian Salmon, Storytelling. La machine à fabriquer les histoires et à formater les esprits, Paris, La Découverte, 2007.

2 Sur ce sujet, voir Francesca Polletta, It Was Like a Fever: Storytelling in Protest and Politics, Chicago, University of 